University of South Carolina

Scholar Commons

$12-2-2010$

\title{
Geometry-Dependent Oxygen Diffusion Flux and Limiting Current Density of the Cathode in a Cathode-Supported Solid Oxide Fuel Cell
}

\author{
Kevin Huang \\ University of South Carolina - Columbia, huang46@cec.sc.edu \\ James L. Shull
}

Follow this and additional works at: https://scholarcommons.sc.edu/emec_facpub

Part of the Mechanical Engineering Commons

\footnotetext{
Publication Info

Published in Journal of The Electrochemical Society, Volume 158, Issue 2, 2010, pages B84-B90.

(C) Journal of The Electrochemical Society 2010, The Electrochemical Society.

(c) The Electrochemical Society, Inc. 2010. All rights reserved. Except as provided under U.S. copyright law, this work may not be reproduced, resold, distributed, or modified without the express permission of The Electrochemical Society (ECS). The archival version of this work was published in Journal of The Electrochemical Society.

Publisher's Version: http://dx.doi.org/10.1149/1.3517456

Huang, K. \& Shull, J. L. (2010). Geometry-Dependent Oxygen Diffusion Flux and Limiting Current Density of the Cathode in a Cathode-Supported Solid Oxide Fuel Cell. Journal of The Electrochemical Society, 158 (2), B84 - B90. http://dx.doi.org/10.1149/1.3517456
}

This Article is brought to you by the Mechanical Engineering, Department of at Scholar Commons. It has been accepted for inclusion in Faculty Publications by an authorized administrator of Scholar Commons. For more information, please contact digres@mailbox.sc.edu. 


\title{
(E) \\ Geometry-Dependent Oxygen Diffusion Flux and Limiting Current Density of the Cathode in a Cathode-Supported Solid Oxide Fuel Cell
}

\author{
Kevin Huang $^{\text {a,*,z }}$ and James L. Shull ${ }^{\mathrm{b}}$ \\ ${ }^{a}$ Department of Mechanical Engineering, University of South Carolina, Columbia, South Carolina 29208, \\ USA \\ ${ }^{b}$ Siemens Energy, Incorporated, Stationary Fuel Cells, Pittsburgh, Pennsylvania 15235, USA
}

\begin{abstract}
An analysis is performed on the diffusion of oxygen through tubular porous cathode substrates having several different geometries. It is shown that the flux of oxygen as it diffuses through these different substrate geometries can be explicitly expressed by a general analytical form with a unique geometric factor for each type of substrate geometry. Experimental measurements of the geometry-independent term, oxygen diffusivity, were conducted for two representative geometries: cylindrical and triangular tubes. These measurements show good agreement between samples with similar porosities and thus favorably support the oxygen flux equations presented. Formulations for the limiting current density were also derived directly from the oxygen flux equations. These are similarly expressed by a general analytical form with the same unique geometric factor depending on the particular substrate. Finally, three representative geometries, cylindrical, triangular, and square tubes, are used to illustrate the influence of the various dimensional parameters on the limiting current density.
\end{abstract}

(C) 2010 The Electrochemical Society. [DOI: 10.1149/1.3517456] All rights reserved.

Manuscript submitted May 28, 2010; revised manuscript received October 26, 2010. Published December 2, 2010.

Porous electrodes are highly desirable in modern solid oxide fuel cell (SOFC) as they are able to provide a large number of electrochemically active sites or triple-phase boundaries where surface adsorption/dissociation reactions and charge transfer of the reactive species can occur. Because the electrode reactions typically occur at or near interfaces with the electrolyte, the gaseous reactants and products must diffuse through the porous structure to get to and from the electrochemically active sites, respectively. In this way the porous electrodes inevitably act as physical barriers to gas molecules, imposing resistance and therefore slowing down the overall rate of mass transport. It is apparent that reduced thickness and/or higher porosity in the porous electrode would help lower the diffusion resistance. Unfortunately, both actions would also make the porous electrode mechanically weaker. This is particularly important for electrode-supported SOFCs where maintaining sufficient mechanical strength in the substrate is imperative. Therefore, the thickness, porosity (sometimes also tortuosity), and mechanical strength of a porous electrode substrate all have to be properly balanced in order to meet both the mechanical and electrical requirements. One simple and common engineering solution is to enhance the mechanical strength by increasing the thickness and reducing the porosity of the electrode substrate to a degree at which the diffusion resistance is still acceptable for achieving the required electrical performance. However, in this compromise, there is a loss of electrical performance. For instance, an earlier study of cathode-supported SOFCs ${ }^{1}$ indicated that the gas-diffusion related concentration polarization of the cathode alone was responsible for more than $25 \%$ of the total voltage losses at a relatively lower current density of $0.30 \mathrm{~A} / \mathrm{cm}^{2}$ and at a relatively high temperature of $950{ }^{\circ} \mathrm{C}$. This is unacceptable for a commercial SOFC product. To find the optimized "sweet spot" for mechanical strength and electrical performance, a study of gas diffusion behavior in porous electrodes and its impact on the electrical performance is essential for technological development and is also important for scientific understanding.

The transport of gaseous species through porous media has been well established in many classical textbooks and review papers. ${ }^{2-5}$ In theory, the Stefan-Maxwell equation is the most general and convenient analytical form for describing mass transport through porous media. $^{2}$ The model takes into account of ordinary molecular diffusion, Knudsen diffusion, and the effect of a finite pressure gradient in multicomponent gas systems. Other mass transport formulations,

\footnotetext{
* Electrochemical Society Active Member

${ }^{\mathrm{z}}$ E-mail: huang46@cec.sc.edu
}

such as the well-known Fick's and dusty-gas models ${ }^{3}$ are essentially derivatives of the Maxwell-Stefan model for special cases. In SOFC modeling, there are a vast number of studies on gas transport phenomena involving either a porous cathode or a porous anode substrate (Refs. 6-10, just to cite a few). It is commonly seen that the Stefan-Maxwell and the dusty-gas models are interchangeably used without noticeable difference in the outcome for the relatively simpler gas systems encountered in SOFCs. ${ }^{11}$ These models have been proven to be effective in analyzing and predicting the performance of porous electrode-supported SOFCs.

Theoretically speaking, the diffusion of oxygen through a porous cathode substrate is intrinsically slower than that of its counterpart hydrogen through a similar anode substrate in electrode-supported SOFCs. In the presence of an operating current, such a fundamental difference gives rise to a higher concentration polarization in the cathode than in the anode. This has become one of the main reasons why the performance of cathode-supported SOFCs is generally lower than that of anode-supported ones. In this paper, the StefanMaxwell model is applied as a fundamental equation to describe the rate of the mass transport of oxygen in air through a number of porous cathode substrates having different geometries. The resultant geometry-dependent oxygen diffusion flux equations are then applied to current flow using Faraday's law, leading to a series of explicit analytical expressions for the limiting current density. Finally, experimental data are used to verify the derived equations for oxygen flux and limiting current density in a porous cathode.

\section{Geometry-Dependent Oxygen Diffusion Flux of the Cathode in an Idealized Shape}

Stefan-Maxwell equation.- The mass transport of a multicomponent gas system under a one-dimensional coordinate can be described by the Stefan-Maxwell model ${ }^{2,5}$ in the following analytical form

$$
\frac{j_{m}}{D_{\mathrm{K}, m}}+\sum_{n=1} \frac{y_{n} j_{m}-y_{m} j_{n}}{D_{m n}}=-\frac{P_{\mathrm{t}}}{R T} \frac{d y_{m}}{d x}
$$

where $j_{m}$ and $j_{n}$ in $\mathrm{mol} /\left(\mathrm{cm}^{2} \mathrm{~s}\right)$ are the molar flux densities of components $m$ and $n$, respectively; $D_{\mathrm{K}, m}$ and $D_{m n}$ are the Knudsen diffusivity for component $m$ and binary diffusivity of $m$ through $n$, respectively; $P_{\mathrm{t}}$ is the total system pressure in atm; $y_{m}$ and $y_{n}$ are the molar fractions of components $m$ and $n$, respectively; $x$ is the distance of diffusion in $\mathrm{cm} ; R\left[82.05\left(\mathrm{~atm} \mathrm{~cm}^{3}\right) /(\mathrm{mol} \mathrm{K})\right]$ and $T$ have their usual meanings. For air, a binary mixture of $\mathrm{O}_{2}-\mathrm{N}_{2}$, Eq. 1 is simplified into 


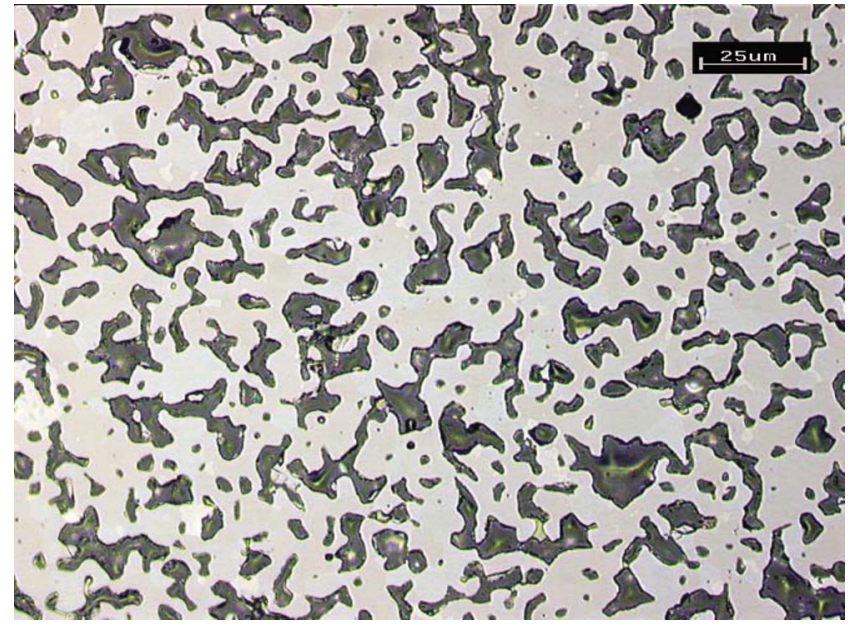

Figure 1. (Color online) A typical microstructure of the porous cathode used in this study.

$$
\frac{j_{\mathrm{O}_{2}}}{D_{\mathrm{K}, \mathrm{O}_{2}}}+\frac{y_{\mathrm{N}_{2}} j_{\mathrm{O}_{2}}-y_{\mathrm{O}_{2}} j_{\mathrm{N}_{2}}}{D_{\mathrm{O}_{2} \mathrm{~N}_{2}}}=-\frac{P_{\mathrm{t}}}{R T} \frac{d y_{\mathrm{O}_{2}}}{d x}
$$

where the partial pressure of oxygen $P_{\mathrm{O}_{2}}=y_{\mathrm{O}_{2}} P_{\mathrm{t}}$.

Compare Eq. 2 with the classical Fick's first law of diffusion for the $\mathrm{O}_{2}$ component

$$
j_{\mathrm{O}_{2}}=-\frac{P_{\mathrm{t}} D_{\mathrm{O}_{2}}}{R T} \frac{d y_{\mathrm{O}_{2}}}{d x}
$$

and with $y_{\mathrm{O}_{2}}+y_{\mathrm{N}_{2}}=1$ and $\mathbf{j}_{\mathrm{N}_{2}}=0$ under a steady-state SOFC operation, it is not difficult to find

$$
D_{\mathrm{O}_{2}}=\left(\frac{1}{D_{\mathrm{K}, \mathrm{O}_{2}}}+\frac{1-y_{\mathrm{O}_{2}}}{D_{\mathrm{O}_{2} \mathrm{~N}_{2}}}\right)^{-1}
$$

Generally speaking, the Knudsen process is significant only at low pressure and small pore diameter. However, there are instances where both Knudsen diffusion and molecular diffusion can be important. Equation 4 implies that Knudsen diffusion and ordinary molecular diffusion compete with one another by a "resistances in parallel" model. For straight cylindrical pores, such a competition can also be conveniently evaluated by the Knudsen number, $\mathrm{Kn}$, which is defined by ${ }^{12}$

$$
\begin{gathered}
K n=\frac{\lambda}{d_{\text {pore }}} \\
\lambda=\frac{k T}{\sqrt{2} \pi \sigma_{\mathrm{A}}^{2} P_{\mathrm{t}}}
\end{gathered}
$$

where $\lambda$ is the mean free path length; $\sigma_{\mathrm{A}}$ is the Lennard-Jones diameter of the spherical molecule. By rule, ordinary molecular diffusion predominates where $K n<1$ whereas Knudsen diffusion predominates where $K n>10$. Application of this rule to the porous cathode substrate of this study shown in Fig. 1 with an average pore size of $10 \mu \mathrm{m}, \sigma_{\mathrm{O}_{2}} \approx 0.3433 \mathrm{~nm}, T=1073 \mathrm{~K}, P_{\mathrm{t}}=101300$ Pa reveals $K n=0.0028$, suggesting Knudsen diffusion in such a substrate is negligible in comparison to ordinary molecular diffusion. Therefore, Knudsen diffusion is not considered in the following discussions.

For the ordinary molecular diffusion of the oxygen in the nitrogen, the oxygen diffusivity, $D_{\mathrm{O}_{2} \mathrm{~N}_{2}}$, as a function of temperature is described by Hirschfelder's equation 12

$$
D_{\mathrm{O}_{2} \mathrm{~N}_{2}}(T)=D_{\mathrm{O}_{2} \mathrm{~N}_{2}}(298) \times\left(\frac{T}{298}\right)^{1.5}
$$

The oxygen diffusivity at $298 \mathrm{~K}, D_{\mathrm{O}_{2} \mathrm{~N}_{2}}(298)$, is used as a reference and can be readily calculated using Lennard-Jones potential theory by

$$
D_{\mathrm{O}_{2} \mathrm{~N}_{2}}(298)=\frac{0.001858 \times(298)^{3 / 2} \times\left[\frac{1}{M_{\mathrm{O}_{2}}}+\frac{1}{M_{\mathrm{N}_{2}}}\right]}{P_{\mathrm{t}} \times \sigma_{\mathrm{O}_{2}}^{2} \times \Omega_{\mathrm{D}}}
$$

where $M_{\mathrm{O}_{2}}$ and $M_{\mathrm{N}_{2}}$ are the molecular weights of $\mathrm{O}_{2}$ and $\mathrm{N}_{2}$ in $\mathrm{g} / \mathrm{mol}$, respectively; $\Omega_{\mathrm{D}}$ is the "collision integral" for molecular diffusion, a dimensionless function of the temperature and of the intermolecular potential-field for one oxygen molecule and one nitrogen molecule. Both $\sigma_{\mathrm{O}_{2}}$ and $\Omega_{\mathrm{D}}$ values can be found in the appendix of Ref. 12. The oxygen diffusivity can be theoretically calculated for any temperatures with Eqs. 7 and 8.

Note that the above relationships of diffusivity are based on diffusion within straight, cylindrical pores aligned in a parallel array. The interaction between gaseous species and the porous body is not considered. However, in most porous materials, pores with various diameters are twisted and interconnected with one another, and the paths for diffusion of gas molecules within the pores are tortuous. For these materials, if an average pore diameter is assumed, a reasonable approximation for the effective diffusivity of a binary gas $\mathrm{AB}$ in random pores is given by

$$
D_{\mathrm{AB}}^{\mathrm{eff}}=\frac{\varepsilon}{\tau} D_{\mathrm{AB}}
$$

where $\varepsilon$ and $\tau$ are porosity and tortuosity, respectively. In some cases, $\tau=1 / \varepsilon$ can be assumed, which leads to

$$
D_{\mathrm{AB}}^{\mathrm{eff}}=\varepsilon^{2} D_{\mathrm{AB}}
$$

From Eq. 10, the porosity can be estimated if the effective diffusivity is measurable.

Ignoring Knudsen diffusion for this study as suggested by the above estimation, the binary Stefan-Maxwell model equation 2 can be further simplified into

$$
j_{\mathrm{O}_{2}}=-\frac{D_{\mathrm{O}_{2} \mathrm{~N}_{2}}^{\mathrm{eff}}}{R T} \frac{d P_{\mathrm{O}_{2}}}{d x}+y_{\mathrm{O}_{2}} j_{\mathrm{O}_{2}}
$$

where $D_{\mathrm{O}_{2} \mathrm{~N}_{2}}^{\text {eff }}$ in $\mathrm{cm}^{2} / \mathrm{s}$ is the effective oxygen diffusivity through the porous cathode substrate, which varies with temperature and microstructure of the substrate. For simplicity, $P_{\mathrm{O}_{2}}$ is denoted by $P$ in the following context.

The connection between the passing electrical current $i\left(\mathrm{~A} / \mathrm{cm}^{2}\right)$ and the incoming oxygen flux density $j_{\mathrm{O}_{2}}$ at steady state in a SOFC is given by Faraday's law

$$
i=4 F j_{\mathrm{O}_{2}}
$$

where $F$ is Faraday's constant, 96,485 C/mol. There are a total of four electrons transferred per oxygen molecule. The importance of Eq. 12 is that it outlines the basic relationship between a mass quantity and an electrical quantity, with which the limiting current density $i_{\mathrm{L}}$ can be evaluated for the concentration polarization of the cathode.

In the following, the above flux equations will be applied to oxygen diffusion through a porous cathode substrate in different geometries.

Planar plate.- The oxygen diffusion in planar geometry is the simplest, one-dimensional process. Figure 2 shows the schematic of oxygen diffusion through a porous cathode plate in dimensions of $t$ (thickness), $s$ (width), and $L$ (length). Rearrange Eq. 11 with using oxygen flux $J_{\mathrm{O}_{2}}=j_{\mathrm{O}_{2}} \times A$ into the following form 


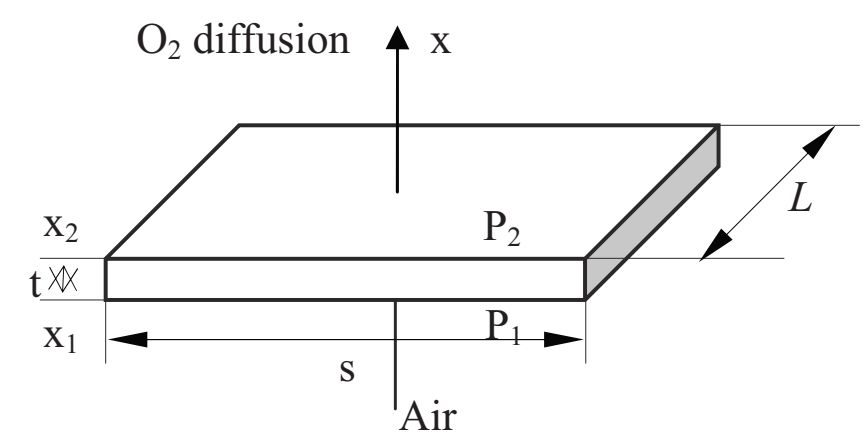

Figure 2. Schematic of oxygen diffusion through a porous planar cathode plate.

$$
J_{\mathrm{O}_{2}} d x=D_{\mathrm{O}_{2} \mathrm{~N}_{2}}^{\mathrm{eff}} \times(s \times L) \times \frac{P_{\mathrm{t}}}{R T} \times \frac{d\left(1-P / P_{\mathrm{t}}\right)}{1-P / P_{\mathrm{t}}}
$$

where the diffusion area $A=s \times L$. At steady state, $J_{\mathrm{O}_{2}}$ is a constant. Integrating $x$ from $x_{1}$ to $x_{2}$ on the left-hand side and $P$ from $P_{1}$ to $P_{2}$ on the right-hand side yields

$$
J_{\mathrm{O}_{2}}=D_{\mathrm{O}_{2} \mathrm{~N}_{2}}^{\text {eff }} \times L \times\left(\frac{s}{t}\right) \times \frac{P_{\mathrm{t}}}{R T} \times \ln \left(\frac{1-P_{2} / P_{\mathrm{t}}}{1-P_{1} / P_{\mathrm{t}}}\right)
$$

where $t=x_{2}-x_{1} ; P_{1}$ and $P_{2}$ are the partial pressures of oxygen at bottom and top surfaces, respectively. The ratio $s / t$ is a dimensionless quantity and defined in this study as the geometric factor $G_{\mathrm{pl}}$ $=s / t$ (pl denotes planar). It is a unique parameter that is dependent on the geometry. Introducing the term "diffusion conductance $K_{\mathrm{pl}}$ "

$$
K_{\mathrm{pl}}=D_{\mathrm{O}_{2} \mathrm{~N}_{2}}^{\text {eff }} \times\left(\frac{s}{t}\right)=D_{\mathrm{O}_{2} \mathrm{~N}_{2}}^{\text {eff }} \times G_{\mathrm{pl}}
$$

and substituting back into Eq. 14 gives

$$
\frac{J_{\mathrm{O}_{2}}}{L}=K_{\mathrm{pl}} \times \frac{P_{\mathrm{t}}}{R T} \times \ln \left(\frac{1-P_{2} / P_{\mathrm{t}}}{1-P_{1} / P_{\mathrm{t}}}\right)
$$

Clearly, Eq. 16 depicts the oxygen flux $(\mathrm{mol} / \mathrm{s})$ per unit length of a porous planar plate. $K_{\mathrm{pl}}$ is a practically important parameter for quality-control in large-volume production of porous substrates. It allows diffusion characteristics of the same kind of substrates at different lengths to be conveniently compared. In what follows, applications of $K$ and $G$ terms will also be extended to other geometries.

Cylindrical tube.- The oxygen diffusion through a porous hollow cylinder as shown in Fig. 3 can be mathematically treated in the direction of radius to allow for an equal oxygen flux at each diffusion front. It is often referred in the textbook as the "diffusion under polar coordinate." Applying Eq. 11 to the geometry with the diffusion area $A=2 \pi r L$ leads to oxygen flux $J_{\mathrm{O}_{2}}$

$$
J_{\mathrm{O}_{2}} d \ln r=\frac{D_{\mathrm{O}_{2} \mathrm{~N}_{2}}^{\mathrm{eff}} P_{\mathrm{t}}}{R T} \times L \times 2 \pi \times \frac{d\left(1-P / P_{\mathrm{t}}\right)}{\left(1-P / P_{\mathrm{t}}\right)}
$$

where $L$ and $r$ are the length and radius of the tube, respectively.

At steady state, integration of $P$ in Eq. 17 with respective to $r$ across the wall of the tube yields

$$
\frac{J_{\mathrm{O}_{2}}}{L}=D_{\mathrm{O}_{2} \mathrm{~N}_{2}}^{\text {eff }} \times \frac{2 \pi}{\ln \left(r_{2} / r_{1}\right)} \times \frac{P_{\mathrm{t}}}{R T} \times \ln \left(\frac{1-P_{2} / P_{\mathrm{t}}}{1-P_{1} / P_{\mathrm{t}}}\right)
$$

where $P_{2}$ and $P_{1}$ are the oxygen concentration at the outer and inner surfaces of the tube, respectively; $r_{2}$ and $r_{1}$ are the outer and inner radii of the tube, respectively. Analogous to Eq. 15, the diffusion conductance of the cylindrical tube, $K_{\mathrm{cyl}}$, is given by

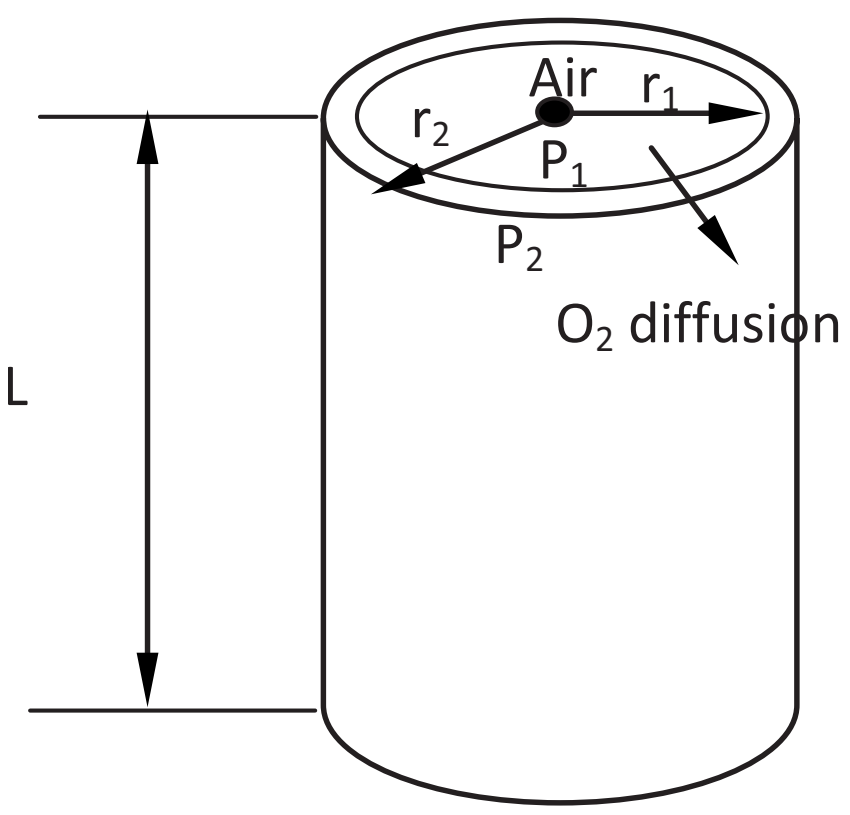

Figure 3. Schematic of oxygen diffusion through a porous cylindrical hollow cathode tube.

$$
K_{\mathrm{cyl}}=D_{\mathrm{O}_{2} \mathrm{~N}_{2}}^{\mathrm{eff}} \times \frac{2 \pi}{\ln \left(r_{2} / r_{1}\right)}=D_{\mathrm{O}_{2} \mathrm{~N}_{2}}^{\mathrm{eff}} \times G_{\mathrm{cyl}}
$$

where the dimensionless geometric factor $G_{\text {cyl }}=2 \pi / \ln \left(r_{2} / r_{1}\right)$. Clearly, thinner wall $\left(t=r_{2}-r_{1}\right)$ and greater $r_{1}$ will increase the diffusion conductance or diffusion capacity of a cylindrical tube.

Rounded triangular tube.- The schematic of an equilateral and rounded triangular tube considered in this study is illustrated in Fig. $4 \mathrm{a}$. The round corners are preferable for reducing the magnitude of thermal stresses induced during thermal transients. The inner and outer curvatures at each corner share the same center. Therefore, the total oxygen flux can be viewed as contributions from two individual sources: round corners and equilateral sides, each of which has been separately discussed in the last two sections. Note that there are a total of three rounded corners and three equilateral sides. Each of the round corners equals to one-third of a full circle as shown in Fig. 3 and each of the side wall represents a planar plate as shown in Fig. 2. Therefore, the total steady-state oxygen flux of the triangular tube, $J_{\mathrm{O}_{2}}^{\mathrm{tri}}$, is given by

$$
\begin{aligned}
\frac{J_{\mathrm{O}_{2}}^{\mathrm{tri}}}{L}= & \frac{J_{\mathrm{O}_{2}}^{\mathrm{cyl}}+J_{\mathrm{O}_{2}}^{\mathrm{pl}}}{L}=D_{\mathrm{O}_{2} \mathrm{~N}_{2}}^{\mathrm{eff}} \times\left(\left(\frac{2 \pi}{\ln \left(r_{2} / r_{1}\right)}\right)+\left(\frac{3 s}{r_{2}-r_{1}}\right)\right) \times \frac{P_{\mathrm{t}}}{R T} \\
& \times \ln \left(\frac{1-P_{2} / P_{\mathrm{t}}}{1-P_{1} / P_{\mathrm{t}}}\right)
\end{aligned}
$$

where $L$ is the length of the triangular tube; $r_{1}$ and $r_{2}$ are the radii of inner and outer corners of the triangular tube, respectively; Clearly, the wall thickness $t=r_{2}-r_{1}$; $s$ is the width of straight section of the equilateral side. In practice, parameter $s$ is not always conveniently available. In order to convert $s$ to other easily measurable parameters, the geometric relationship of the triangular tube is further elaborated in Fig. 4b. The two easily measurable parameters, such as the heights of the inner and outer triangles, $H$ and $h$, respectively, have the following geometric relationship with parameter $s$

$$
s=\frac{2 \sqrt{3}}{3}\left(H-2 r_{2}\right)
$$




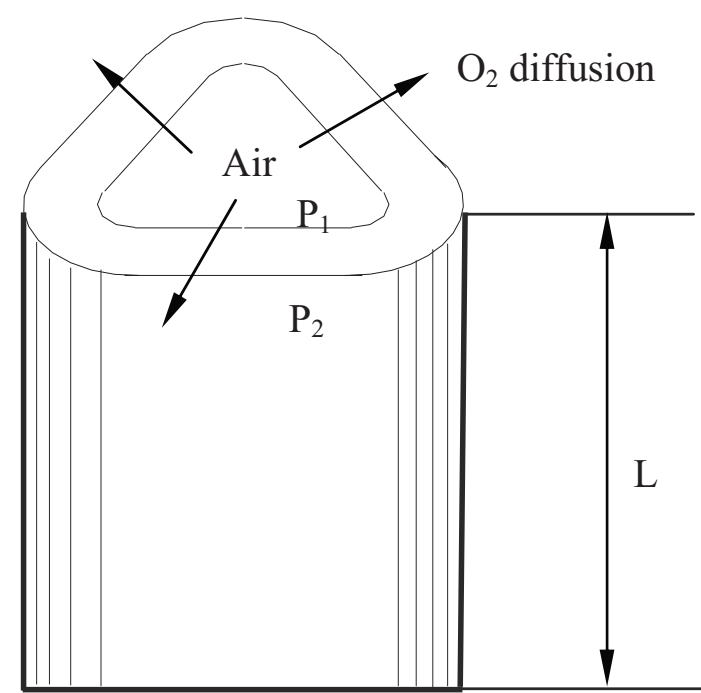

(a)

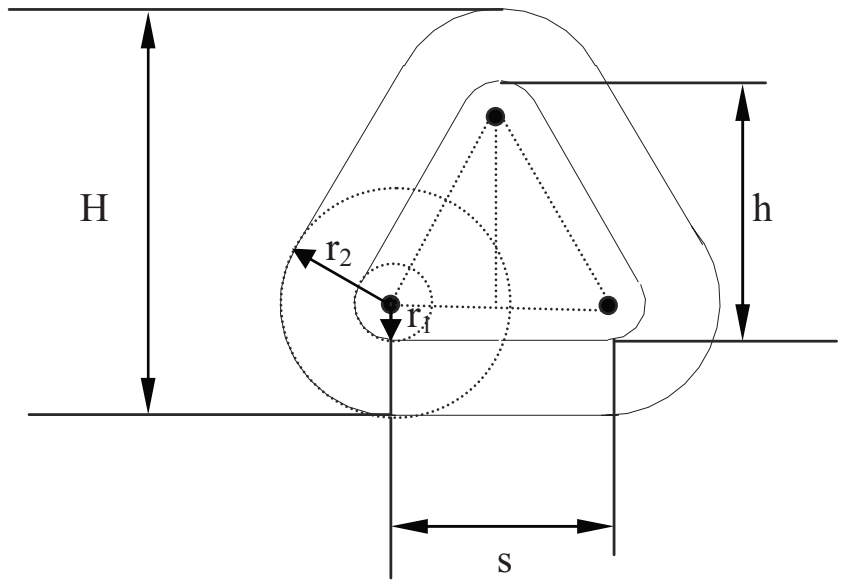

(b)

Figure 4. (a) Oxygen diffusion process in an idealized porous triangular cathode tube and (b) cross section.

$$
s=\frac{2 \sqrt{3}}{3}\left(h-2 r_{1}\right)
$$

The diffusion conductance $K_{\text {tri }}$ is written from Eq. 20

$$
K_{\mathrm{tri}}=D_{\mathrm{O}_{2} \mathrm{~N}_{2}}^{\mathrm{eff}} \times\left(\frac{2 \pi}{\ln \left(r_{2} / r_{1}\right)}+\frac{2 \sqrt{3}\left(H-2 r_{2}\right)}{r_{2}-r_{1}}\right)=D_{\mathrm{O}_{2} \mathrm{~N}_{2}}^{\mathrm{eff}} \times G_{\text {tri }}
$$

Evidently, the diffusion conductance (or diffusion capacity) of a triangular tube can be increased by reducing the wall thickness, increasing the inner radius of the corners, and making the triangle larger.

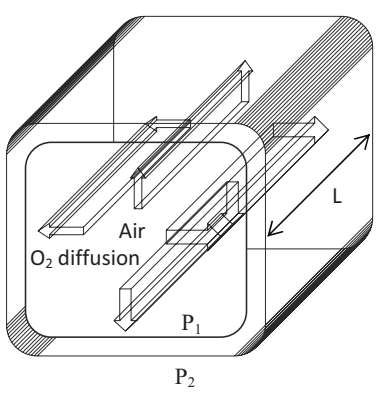

(a)

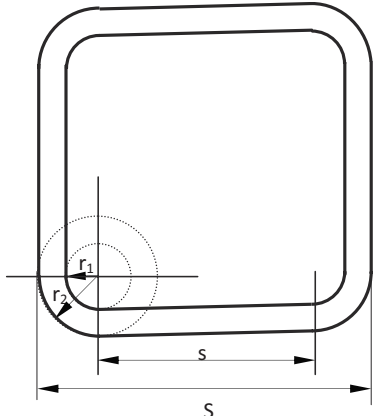

(b)
Figure 5. (a) Oxygen diffusion process in an idealized porous square cathode tube and (b) cross section.

Rounded square tube.- The schematic of a rounded square tube $^{\mathrm{c}}$ is shown in Fig. 5, where $r_{1}$ and $r_{2}$ are the radii of the inner and outer corners, respectively, which share the same center; $S$ is the width of the square; $L$ is the length of the tube. Clearly, the wall thickness $t=r_{2}-r_{1}$. Following the same procedure as previously described for the triangular tube, the steady-state oxygen flux is given by

$$
\begin{aligned}
\frac{J_{\mathrm{O}_{2}}^{\mathrm{sqr}}}{L}= & \frac{J_{\mathrm{O}_{2}}^{\mathrm{cyl}}+J_{\mathrm{O}_{2}}^{\mathrm{pl}}}{L}=D_{\mathrm{O}_{2} \mathrm{~N}_{2}}^{\mathrm{eff}} \times\left(\left(\frac{2 \pi}{\ln \left(r_{2} / r_{1}\right)}\right)+\left(\frac{4 s}{r_{2}-r_{1}}\right)\right) \times \frac{P_{\mathrm{t}}}{R T} \\
& \times \ln \left(\frac{1-P_{2} / P_{\mathrm{t}}}{1-P_{1} / P_{\mathrm{t}}}\right)
\end{aligned}
$$

Note that there are a total of four round corners and four equilateral sides. Each round corner represents a quadrant of a full circle and each side is similar to a planar plate. The partial width, $s$, through which the planar diffusion take place, has the following geometric relationship with the easily measurable full width, $S$, of the square

$$
s=S-2 r_{2}
$$

The diffusion conduction $K_{\text {sqr }}$ is then written from Eq. 23 as

$$
K_{\mathrm{sqr}}=D_{\mathrm{O}_{2} \mathrm{~N}_{2}}^{\text {eff }} \times\left(\frac{2 \pi}{\ln \left(r_{2} / r_{1}\right)}+\frac{4\left(S-2 r_{2}\right)}{r_{2}-r_{1}}\right)=D_{\mathrm{O}_{2} \mathrm{~N}_{2}}^{\text {eff }} \times G_{\text {sqr }}
$$

It is evident that the diffusion conductance of a rounded square tube can be enhanced by those factors that would similarly increase the diffusion conductance of a triangular tube.

In summary, the steady-state oxygen diffusion fluxes of different geometries follow a general analytical form

$$
\frac{J_{\mathrm{O}_{2}}}{L}=K \times \frac{P_{\mathrm{t}}}{R T} \times \ln \left(\frac{1-P_{2} / P_{\mathrm{t}}}{1-P_{1} / P_{\mathrm{t}}}\right)
$$

The differences are only reflected in the diffusion conductance $K$, more specifically, the geometric factor, $G$, for representing different geometries. In the following, the derived oxygen flux equations will be further connected with the electrical current for which it supports via the charge-transfer process. This will lead to the limiting current or current density to be discussed below.

Validation of the models. - To validate the above diffusion models, the geometry-independent $D_{\mathrm{O}_{2} \mathrm{~N}_{2}}^{\text {eff }}$ values of two cathode substrates with the same microstructure but in different geometries will be compared. If the obtained $D_{\mathrm{O}_{2} \mathrm{~N}_{2}}^{\text {eff }}$ values at a given porosity are in

\footnotetext{
${ }^{c}$ The rectangular tube can also be treated similarly with two different side widths.
} 


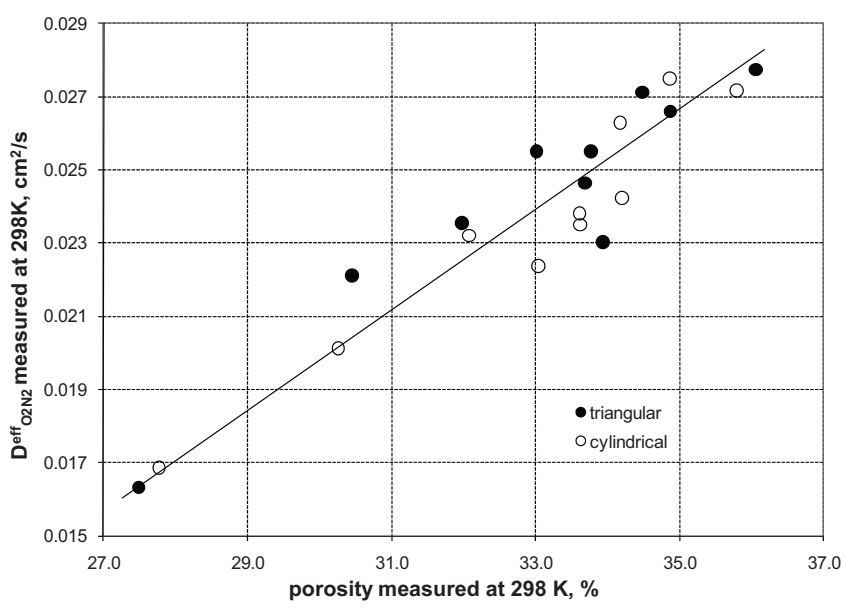

Figure 6. Comparison of oxygen diffusivity measured from porous cylindrical and triangular cathode tubes. Courtesy of Stationary Fuel Cells, Siemens Energy, Inc.

good agreement, the models are considered validated. In this study, the geometries of cylindrical tube and triangular tube are selected for the comparison.

The attainment of $D_{\mathrm{O}_{2} \mathrm{~N}_{2}}^{\text {eff }}$ for cylindrical and triangular cathode tubes can be realized using Eqs. 18 and 20, but requires the knowledge of geometric factors $G$ and oxygen fluxes $J_{\mathrm{O}_{2}}$. While $G$ can be easily calculated with known dimensions, such as length, inner and outer radii, and height of the triangle, $J_{\mathrm{O}_{2}}$ can only be obtained via measurement. The detail on how to measure $J_{\mathrm{O}_{2}}$ of a porous cathode substrate has been described in Ref. 13. The key is to measure the steady-state oxygen concentration in the sweeping gas $\mathrm{N}_{2}$. To achieve this goal, a commercial oxygen analyzer Ametek CG-1000 (Pittsburgh, Pennsylvania) was employed at the downstream of the sweeping gas $\mathrm{N}_{2}$. With the known mass flow rate of the sweeping gas and measured oxygen concentration, $J_{\mathrm{O}_{2}}$ can be given as the product of the two. Independently, the porosity of the same porous substrate was measured by Archimedes' method. Reference 13 also gives a detailed description of the method. For each measurement, five samples were typically used to ensure the repeatability. Figure 6 shows a plot of the averaged $D_{\mathrm{O}_{2} \mathrm{~N}_{2}}^{\text {eff }}$ as a function of the porosity for the two geometries. Within the porosity range plotted, the relationship appears to be linear, not as suggested by Eq. 10. Nevertheless, the agreement in $D_{\mathrm{O}_{2} \mathrm{~N}_{2}}^{\text {eff }}$ of the two geometries favorably supports the oxygen flux models. The effect of tortuosity of the two substrates on the results shown in Fig. 6 can be ignored because the two tubes are fabricated by the same process.

\section{Geometry-Dependent Limiting Current Density of the Cathode in a Cathode-Supported SOFC}

In this section, Faraday's law (Eq. 12) will be used as the fundamental equation to correlate a passing current $I$ with a supplying oxygen flux $J_{\mathrm{O}_{2}}$ across the diffusion interface. In kinetics terms, Eq. 12 requires that the rate of charge transfer (charge per unit time) be matched by the rate of oxygen diffusion. At a critical point where the electrical current can no longer be supported by a steady supply of oxygen molecules due to a limited rate of oxygen diffusion, the current will become saturated. Under such a circumstance, the oxygen at the interface will become depleted, e.g., $P_{2}=0$. The saturated current, which is often known as the limiting current $I_{\mathrm{L}}$ or limiting current density $i_{\mathrm{L}}$, is the subject of the following discussion.

Another important aspect in discussing the limiting current is the consideration of the geometry that is involved in active oxygen diffusion in the cathode substrate of a SOFC. It is very common that the active cathode geometries of a practical SOFC deviate from aforementioned idealized geometries, due to the presence of other

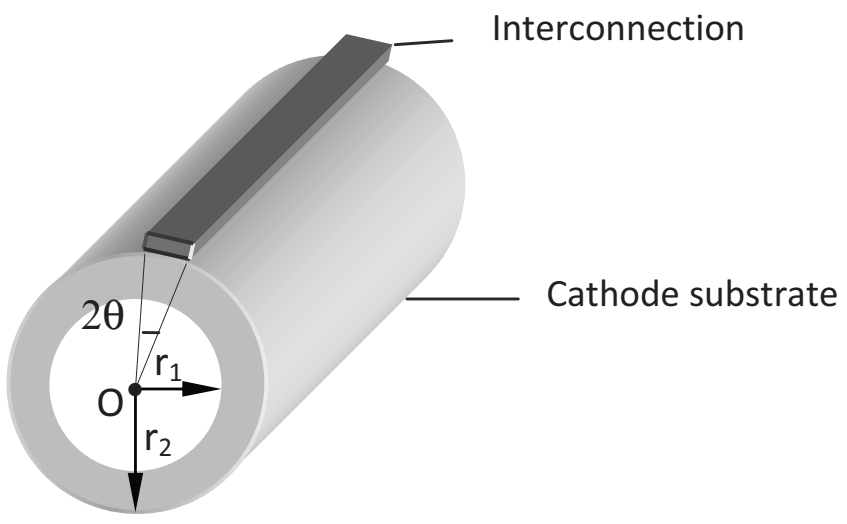

Figure 7. Schematic of a practical porous cylindrical cathode tube with the presence of a dense interconnection layer that is inactive to the oxygen diffusion.

functional layers on the substrate. For these cases, limiting current density instead of limiting current is a better suited quantity to describe the concentration polarization of the cathode.

Planar substrate.- A planar, porous cathode substrate is generally used in the cathode-supported planar SOFCs. No deviation in diffusion area from previous discussion in planar plate exists for this simple geometry. Therefore, the steady-state electrical current $I$ as described by Faraday's law 12 is given by

$$
I=4 F \times D_{\mathrm{O}_{2} \mathrm{~N}_{2}}^{\mathrm{eff}} \times L \times G_{\mathrm{pl}} \times \frac{P_{\mathrm{t}}}{R T} \times \ln \left(\frac{1-P_{2} / P_{\mathrm{t}}}{1-P_{1} / P_{\mathrm{t}}}\right)
$$

When $P_{2}=0$, it leads to the limiting current $I_{\mathrm{L}}$

$$
I_{L}=-\frac{4 F D_{\mathrm{O}_{2} \mathrm{~N}_{2}}^{\mathrm{eff}} P_{\mathrm{t}}}{R T} \times L \times\left(\frac{a}{t}\right) \times \ln \left(1-P_{1} / P_{\mathrm{t}}\right)
$$

Because the oxygen diffusion area is $L \times a$, and the limiting current density $i_{\mathrm{L}}$ becomes

$$
i_{\mathrm{L}}=-\frac{4 F D_{\mathrm{O}_{2} \mathrm{~N}_{2}}^{\mathrm{eff}} P_{\mathrm{t}}}{R T t} \times \ln \left(1-P_{1} / P_{\mathrm{t}}\right)
$$

It is interesting to note that $i_{\mathrm{L}}$ is proportional to $D_{\mathrm{O}_{2} \mathrm{~N}_{2}}^{\text {eff }} \times P_{\mathrm{t}}$ or $D_{\mathrm{O}_{2} \mathrm{~N}_{2}} \times P_{\mathrm{t}}$ at a fixed temperature. For many binary gas mixtures at low pressure $(<30 \mathrm{~atm})$, this product is constant. ${ }^{2}$ Therefore, it is reasonable to postulate that the system pressure $P_{\mathrm{t}}$ has no effect on the concentration polarization of the cathode of most operational SOFCs.

Cylindrical tubular substrate. - The actual oxygen diffusion area in a cylindrical cathode tube such as that used in Siemens/ Westinghouse SOFCs deviated from the discussion in Cylindrical Tube is illustrated in Fig. 7. A portion of the cathode cylinder has to be reserved for the layer of a dense interconnection, which is not active in oxygen diffusion. Since the effective oxygen diffusion area is $L \times(2 \pi-2 \theta) \times r$, a series of actions by integrating Eq. 17, combining with Eq. 12 and letting $P_{2}=0$ lead to the limiting current $I_{\mathrm{L}}$

$$
I_{\mathrm{L}}=-\frac{4 F D_{\mathrm{O}_{2} \mathrm{~N}_{2}}^{\mathrm{eff}} P_{\mathrm{t}}}{R T} \times L \times \frac{2 \pi-2 \theta}{\ln \left(r_{2} / r_{1}\right)} \times \ln \left(1-P_{1} / P_{\mathrm{t}}\right)
$$

where $2 \theta$ is the radian covering the circular portion occupied by the interconnection. The ideal $G$-factor in Eq. 19 is then changed to the effective $G$-factor 


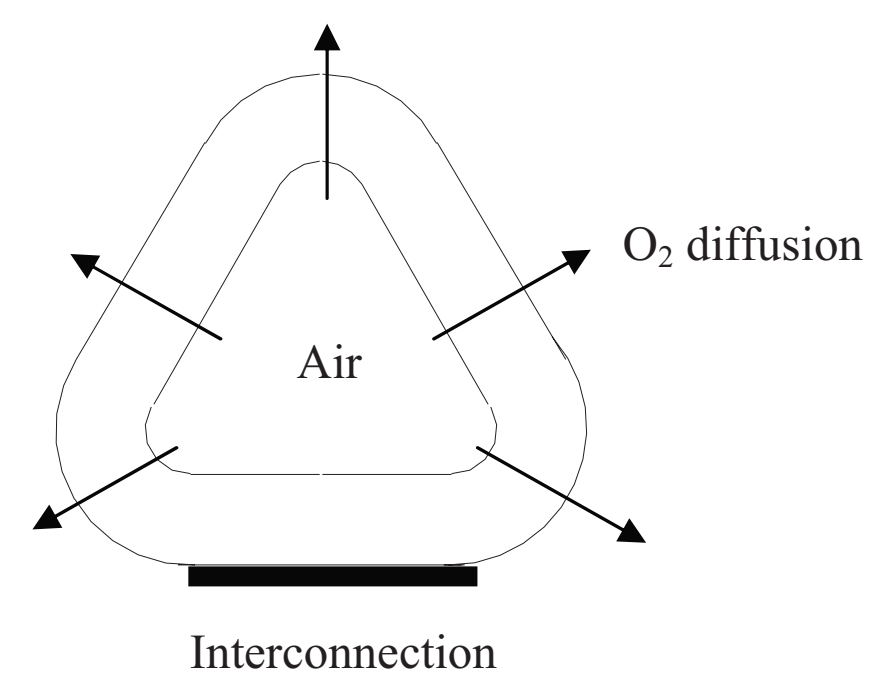

Figure 8. Cross section of a practical porous triangular cathode tube with the presence of a dense interconnection layer that is inactive to the oxygen diffusion.

$$
G_{\mathrm{cyl}}^{\mathrm{eff}}=\frac{2 \pi-2 \theta}{\ln \left(r_{2} / r_{1}\right)}
$$

Dividing Eq. 31 by the active diffusion area $L \times(2 \pi-2 \theta) \times r_{2}$ ( $r_{2}$ is used because it is where $P_{2}=0$ ) leads to the limiting current density $i_{\mathrm{L}}$

$$
i_{\mathrm{L}}=-\frac{4 F D_{\mathrm{O}_{2} \mathrm{~N}_{2}}^{\mathrm{eff}} P_{\mathrm{t}}}{R T} \times \frac{1}{r_{2} \ln \left(r_{2} / r_{1}\right)} \times \ln \left(1-P_{1} / P_{\mathrm{t}}\right)
$$

Note that $i_{\mathrm{L}}$ is no longer a function of $2 \theta$. As aforementioned, the linear dependence of $i_{\mathrm{L}}$ on $D_{\mathrm{O}_{2} \mathrm{~N}_{2}} \times P_{\mathrm{t}}$ suggests that the concentration polarization is most likely not affected by the system pressure $P_{\mathrm{t}}$. This is a valid statement for any geometry discussed in this study.

Triangular tubular substrate.- Similar to the cylindrical tubular SOFCs, a small portion of the triangular cathode tube intended for interconnection is not involved in active oxygen diffusion in a cathode-supported triangular tubular SOFC. This inactive portion of oxygen diffusion is chosen as one side of the triangle. Figure 8 shows a schematic of the interconnection-present triangular cathode tube. A good example is the latest Siemens Delta 8 SOFC design. ${ }^{14}$ In this case, the total oxygen flux $J_{\mathrm{O}_{2}}$ is reduced to the contributions from two sides and three corners. Following the same procedure as described in Rounded Triangular Tube, it leads to the following effective oxygen flux

$$
\frac{J_{\mathrm{O}_{2}}^{\mathrm{tri}}}{L}=\frac{D_{\mathrm{O}_{2} \mathrm{~N}_{2}}^{\text {eff }} P_{\mathrm{t}}}{R T} \times\left(\left(\frac{2 \pi}{\ln \left(r_{2} / r_{1}\right)}\right)+\left(\frac{2 s}{r_{2}-r_{1}}\right)\right) \times \ln \left(\frac{1-P_{2} / P_{\mathrm{t}}}{1-P_{1} / P_{\mathrm{t}}}\right)
$$

The effective oxygen diffusion area is $L \times(2 \pi r+2 s)$. By setting $P_{2}=0$ in Eq. 33, it leads to the limiting current

$$
I_{\mathrm{L}}=-\frac{4 F D_{\mathrm{O}_{2} \mathrm{~N}_{2}}^{\text {eff }} P_{\mathrm{t}}}{R T} \times L \times\left(\frac{2 \pi}{\ln \left(r_{2} / r_{1}\right)}+\frac{2 s}{r_{2}-r_{1}}\right) \times \ln \left(1-P_{1} / P_{\mathrm{t}}\right)
$$

with the effective $G$-factor expressed as

$$
G_{\text {tri }}^{\mathrm{eff}}=\frac{2 \pi}{\ln \left(r_{2} / r_{1}\right)}+\frac{2 s}{r_{2}-r_{1}}
$$

Because the active diffusion area is $L \times\left(2 \pi r_{2}+2 s\right)$ at $P_{2}=0$, the corresponding limiting current density $i_{\mathrm{L}}$ becomes

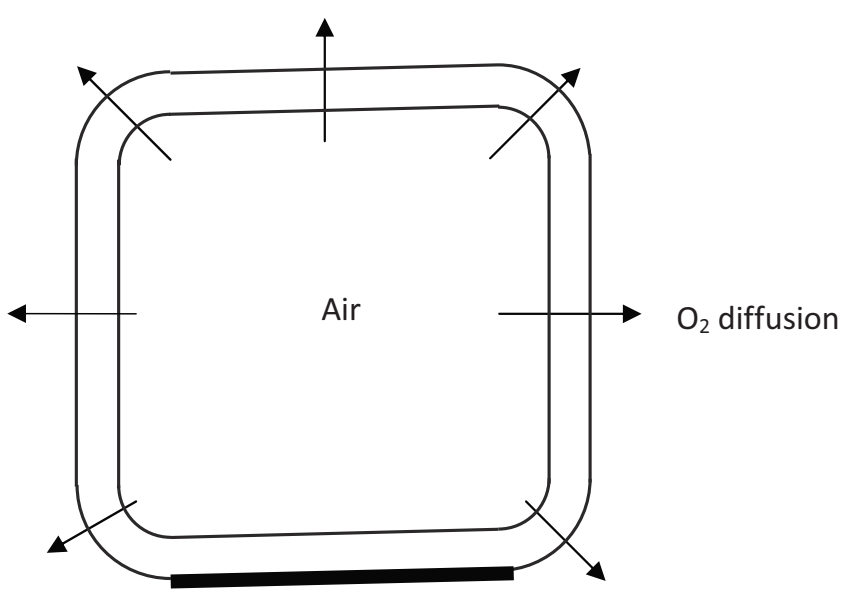

Interconnection

Figure 9. Cross section of a practical porous cathode square tube with the presence of a dense interconnection layer that is inactive to the oxygen diffusion.

$$
i_{\mathrm{L}}=-\frac{4 F D_{\mathrm{O}_{2} \mathrm{~N}_{2}}^{\mathrm{eff}} P_{\mathrm{t}}}{R T} \times \frac{G_{\mathrm{tri}}^{\mathrm{eff}}}{2 \pi r_{2}+2 s} \times \ln \left(1-P_{1} / P_{\mathrm{t}}\right)
$$

Square tubular substrate.-An analogy to the cathodesupported triangular tubular SOFC is to apply the oxygen diffusion inactive interconnection layer to one side of the square tube (see Fig. 9). Therefore, only three sides and four corners involve in the active oxygen diffusion. A close example is the Siemens/Westinghouse HPD SOFC design. Following the same procedure as described in Rounded square tube, the effective oxygen flux is given by

$$
\frac{J_{\mathrm{O}_{2}}^{\mathrm{gqr}}}{L}=\frac{D_{\mathrm{O}_{2} \mathrm{~N}_{2}}^{\text {eff }} P_{\mathrm{t}}}{R T} \times\left(\left(\frac{2 \pi}{\ln \left(r_{2} / r_{1}\right)}\right)+\left(\frac{3 s}{r_{2}-r_{1}}\right)\right) \times \ln \left(\frac{1-P_{2} / P_{\mathrm{t}}}{1-P_{1} / P_{\mathrm{t}}}\right)
$$

The effective oxygen diffusion area is $L \times(2 \pi r+3 s)$. By setting $P_{2}=0$, it leads to the limiting current $I_{\mathrm{L}}$

$$
I_{\mathrm{L}}=-\frac{4 F D_{\mathrm{O}_{2} \mathrm{~N}_{2}}^{\mathrm{eff}} P_{\mathrm{t}}}{R T} \times L \times\left(\frac{2 \pi}{\ln \left(r_{2} / r_{1}\right)}+\frac{3 s}{r_{2}-r_{1}}\right) \times \ln \left(1-P_{1} / P_{\mathrm{t}}\right)
$$

The effective $G$-factor is reduced from Eq. 25 to

$$
G_{\mathrm{sqr}}^{\mathrm{eff}}=\frac{2 \pi}{\ln \left(r_{2} / r_{1}\right)}+\frac{3 s}{r_{2}-r_{1}}
$$

Since the active oxygen diffusion area is $L \times\left(2 \pi r_{2}+3 s\right)$ at $P_{2}$ $=0$, the limiting current density $i_{\mathrm{L}}$ is

$$
i_{\mathrm{L}}=-\frac{4 F D_{\mathrm{O}_{2} \mathrm{~N}_{2}}^{\text {eff }} P_{\mathrm{t}}}{R T} \times \frac{G_{\mathrm{sqr}}^{\mathrm{eff}}}{2 \pi r_{2}+3 s} \times \ln \left(1-P_{1} / P_{\mathrm{t}}\right)
$$

In summary, the limiting current densities of the cathode in different geometries follow a general form

$$
i_{\mathrm{L}}=-\frac{4 F D_{\mathrm{O}_{2} \mathrm{~N}_{2}}^{\text {eff }} P_{\mathrm{t}}}{R T} \times N \times \ln \left(1-P_{1} / P_{\mathrm{t}}\right)
$$

where $N$ represents a geometry-dependent factor. It is important to stress that $i_{\mathrm{L}}$ or $I_{\mathrm{L}}$ is not affected by the system pressure for most SOFC applications.

Applications.— To demonstrate how $i_{\mathrm{L}}$ varies with $D_{\mathrm{O}_{2} \mathrm{~N}_{2}}^{\text {eff }}$ for a given geometry, Fig. 10 compares the $i_{\mathrm{L}}$ of a cylindrical, triangular, and square cathode tubes calculated from Eqs. 32, 36, and 40, re- 


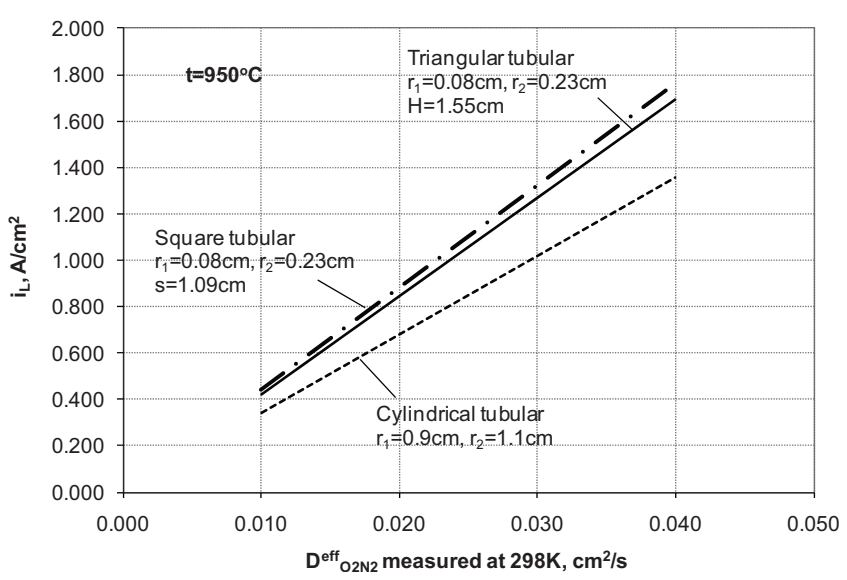

Figure 10. Comparison of limiting current density of porous cylindrical and triangular cathode tubes calculated from Eqs. 25 and 29 for $950{ }^{\circ} \mathrm{C}$.

spectively. The dimensions of the three types of tubes were taken as close to those representing Siemens/Westinghouse designs. It appears that the square tube exhibits a slightly higher $i_{\mathrm{L}}$ than the triangular tube at a given $D_{\mathrm{O}_{2} \mathrm{~N}_{2}}^{\text {eff }}$. However, both of them clearly have the advantage over the cylindrical one over the entire $D_{\mathrm{O}_{2} \mathrm{~N}_{2}}^{\text {eff }}$ range plotted. The enhancement of $i_{\mathrm{L}}$ by the geometry becomes even greater at higher $D_{\mathrm{O}_{2} \mathrm{~N}_{2}}^{\text {eff }}$. The underlying reason for the observation is obviously the differences in the $N$-values shown in Eq. 41 for different geometries. With the dimensions given in Fig. 10, the $\mathrm{N}$-values for the triangular and square tubes are calculated to be 5.74 and 5.89, respectively, whereas the $N$-value of the cylindrical tubes is 4.50. A geometry with a higher $N$-value yields a higher $i_{\mathrm{L}}$.

\section{Summary}

The molecular oxygen diffusion process through a porous cathode substrate was analyzed for several geometries including planar plate, cylindrical tube, triangular tube, and square tube. It was shown that the oxygen flux can be analytically expressed in a general form for these geometries with including a simple geometry identifier (or geometrical factor). A comparison of the independently measured oxygen flux and porosity between cylindrical and triangular tubes indicated a close agreement in oxygen diffusivity, a geometry-independent quantity, at a given porosity. This finding fa- vorably supports the diffusion model proposed. By Faraday's law, the limiting current density was connected with the oxygen flux, from which a series of equations of limiting current or current density have been developed for the geometries discussed. Because there are other functional layers inactive to molecular oxygen diffusion on the cathode substrate, the effective oxygen fluxes in practical SOFCs are generally reduced. A series of limiting current density equations for different cathode geometries have also been generalized into a common form with the geometry-dependent $N$-factor as the identifier. It is important to note that neither oxygen flux density nor limiting current density will be affected by the system pressure because the product of $D_{\mathrm{O}_{2} \mathrm{~N}_{2}}^{\text {eff }} \times P_{\mathrm{t}}$, to which the oxygen flux and limiting current density are proportional, is usually a constant at low pressure $\left(P_{\mathrm{t}}<30 \mathrm{~atm}\right)$.

\section{Acknowledgments}

Part of this work was carried out in Stationary Fuel Cell Division of Siemens Energy. The authors thank the U.S. Department of Energy for financial support of the project under the agreement of DE-FC26-05NT42613 (SECA program) during the period.

University of South Carolina assisted in meeting the publication costs of this article.

\section{References}

1. K. Huang, in Proceedings of the 7th European Solid Oxide Fuel Cell Forum, Lucerne, Switzerland, P0303 (2006).

2. R. B. Bird, W. E. Stewart, and E. N. Lightford, Transport Phenomena, John Wiley \& Sons, New York (1960).

3. E. A. Mason and J. A. Malinauskas, Transport in Porous Media: The Dusty-Gas Model, Elsevier, New York (1983).

4. G. F. Froment and K. B. Bischoff, Chemical Reactor Analysis and Design, John Wiley \& Sons, New York (1990).

5. R. Krishna and J. A. Wesselingh, Chem. Eng. Sci., 52, 861 (1997)

6. J. W. Kim, A. V. Virkar, K. Z. Fung, K. Mehta, and S. C. Singhal, J. Electrochem. Soc., 146, 69 (1999).

7. S. H. Chan, K. A. Khor, and Z. T. Xia, J. Power Sources, 93, 130 (2001).

8. S. H. Chan and Z. T. Xia, J. Electrochem. Soc., 148, A388 (2001).

9. S. Nagata, A. Momma, T. Kato, and Y. Kasuga, J. Power Sources, 101, 60 (2001).

10. M. Iwata, T. Hikosaka, M. Morita, T. Iwanari, K. Ito, K. Onda, Y. Esaki, Y. Sakaki, and S. Nagata, Solid State Ionics, 132, 297 (2000).

11. R. Suwanwarangkul, E. Croiset, M. W. Fowler, P. L. Douglas, E. Entchev, and M. A. Douglas, J. Power Sources, 122, 9 (2003).

12. J. R. Welty, C. E. Wicks, R. E. Wilson, and G. Rorrer, Fundamentals of Momentum, Heat, and Mass Transfer, 4th ed., p. 431, John Wiley \& Sons, New York (2001).

13. K. Huang and J. B. Goodenough, Solid Oxide Fuel Cell Technology: Principles, Performance and Operations, pp. 179-182, Woodhead Publishing Co., Cambridge, UK (2009).

14. K. Huang, ECS Trans., 12(1), 375 (2007). 